\title{
Thrombotic Microangiopathy Induced by Hyperthermal Intraperitoneal Chemotherapy with Mitomycin C
}

\author{
Mahir Köksal ${ }^{1}$, Robert J. van Ginkel ${ }^{\mathbf{2}}$, Jan G. Zijlstra ${ }^{\mathbf{1}}$ \\ ${ }^{1}$ Department of Critical Care, University Medical Center Groningen, University of Groningen, Groningen, Netherlands \\ ${ }^{2}$ Department of Surgery, University Medical Center Groningen, University of Groningen, Groningen, Netherlands \\ E-mail: j.g.zijlstra@icv.umcg.nl \\ Received January 26, 2011; revised February 21, 2011; accepted February 28, 2011
}

\begin{abstract}
A patient with pseudomyxoma peritonei underwent Hyperthermal Intraperitoneal Chemotherapy (HIPEC) with Mitomycin C after which she developed thrombotic microangiopathy. This syndrome mimicked possible surgical complications. Treatment with plasma exchange resolved the syndrome and the patient recovered completely. This is the first description of thrombotic microangiopathy early after a single dose intraperitoneal Mitomycin C.
\end{abstract}

Keywords: HIPEC, Toxicity, HUS-TTP

\section{Introduction}

Hyperthermal Intraperitoneal Chemotherapy (HIPEC) is an established therapy in peritoneal malignancies and advocated as standard therapy in pseudomyxoma peritonei. [1,2] With careful selection and optimal perioperative care morbidity and mortality are comparable to other major gastrointestinal surgery. [3] Mortality is mainly due to sepsis and multi-orgaan failure as a result of surgical complications. Systemic toxicity of HIPEC also occurs in about $20 \%$ and is mainly haematological or renal toxicity. [3] We describe a potentially lethal but most times curable complication of HIPEC that can easily be mistaken for a surgical complication or haematological toxicity.

A 65-year old woman was admitted to the ICU for postoperative care after abdominal surgery with Hyperthermal Intraperitoneal Chemotherapy (HIPEC). She had a pseudomyxoma peritonei with a primary tumour in the appendix. Peritonectomy, splenectomy, cholecystectomy, hemicolectomy (cecum and colon ascendens), appendectomy, hysterectomy, bilateral salpingectomy, resection of both the omentum majus and omentum minus, diathermia of the liver capsula, and stripping of both hemidiaphragmata and the pelvic peritoneum was performed. She received HIPEC with a total Mitomycine-C (MMC) dose of $63 \mathrm{mg}$.

On admission to the ICU she was haemodynamically and respiratory stable. She remained somnolent and feb- rile but could be extubated the same day. Four days postoperatively she developed oliguric renal failure with sUrea $20.4 \mathrm{mmol} / \mathrm{l}$ (normal range 2.5 - $7.5 \mathrm{mmol} / \mathrm{l}$ ) and sCreatinin $213 \mu \mathrm{mol} / \mathrm{l}$ (normal range 0 - $90 \mu \mathrm{mol} / \mathrm{l}$ ) levels). Lactate Dehydrogenase (LDH) increased to $1653 \mathrm{U} / \mathrm{l}$ (normal range $0-250 \mathrm{U} / \mathrm{l}$ ) and a marked thrombocytopenia developed (39 × 109 E/l; normal range $150-350 \times$ $109 \mathrm{E} / \mathrm{l})$. Schizocytes were highly positive and haptoglobulin decreased $(<0,2 \mathrm{~g} / \mathrm{l}$; normal range $0.3-2.0 \mathrm{~g} / \mathrm{l})$. Coagulation tests were normal and Coombs test was negative. Blood cultures were negative. ADAMTS13 activity was $45 \%$ (normal range $30 \%-200 \%$ ). The diagnosis atypical haemolytic uremic syndrome, a disorder from the wide spectrum of the thrombotic microangiopathy (TMA) syndrome, was made. Immediate treatment with twice daily plasma exchange with $40 \mathrm{ml}$ Fresh Frozen Plasma (FFP) per kilogram body weight was started. Sixteen days after admission to our department, she could be transferred to the ward in an alert, stable condition with normal thrombocytes and regained renal function. She was readmitted once to the ICU because of sick sinus syndrome requiring a pacemaker. There were no signs of TMA.

TMA is a disorder characterized by haemolytic anaemia, thrombocytopenia, purpura, renal failure and neurological impairment. [4] It is life-threatening; left untreated the mortality rate is up to $95 \%$. The central pathophysiological feature is the formation of hyaline thrombi resulting in hemolysis and micro infarction in 
several organs. The underlying mechanism is a dysbalance between von Willebrand factor (vWf) and von Willebrand processing factor. [5] A local increase in vWf is seen in classic haemolytic uremic syndrome (HUS), or hamburger disease, where thrombi are mainly formed in the kidney, resulting in renal failure and minimal systemic symptoms. In familiar thrombotic thrombocytopenic purpura (TTP) the processing factor ADAMTS13 is decreased and systemic symptoms prevail. Between these two ends of the spectrum there is a range of clinical presentations. Cause, genetic make-up of the patient and many other factors determine the presenting symptoms. Causes are mainly infectious diseases and medication related. The therapy is based on supply of vWf processing factor by FFP. To supply enough FFP plasma exchange is required. In this patient MMC is the most probable cause. [6] Our patient presented with haemolytic anaemia, thrombocytopenia, renal failure and somnolence. The later developing sick sinus can also be due to micro infarction of the heart. After excluding sepsis, immune mediated hemolysis, and disseminated intravascular coagulation the diagnosis atypical HUS was made. [6] MMC induced TMA has been described after intravenous dosing. [4,7-9] It has been suggested that the chance of developing TMA is related to the cumulative dose. Below 36mg/m2 MMC no TMA is seen. [10] The mechanism is not clear although direct endothelial damage has been suggested. [4,7,11] MMC induced TMA has never been described after a single HIPEC procedure. We have no serum levels of MMC but considerable absorption is possible because of the large wound surface created by the extensive surgery. However, systemic toxicity was limited because no toxic leucopenia occurred. MMC induced TMA is considered a late complication because toxicity has mainly been described after repeated doses reaching the cumulative dose after weeks or months. Our patient received more than the suggested maximal dose in a single gift presuming that limited absorption from the abdomen would occur. Our patient shows several other unique features. TMA occurred immediately after a single intraperitoneal dose and responded well to therapy. [6]

Patients treated with HIPEC are prone to surgical complications and to systemic toxicity of the chemotherapy. [3] Both have many symptoms in common with TMA syndrome. Subscribing these symptoms to toxicity or complications is imminent and a potentially lethal but, when recognized, treatable disease can easily be missed.

\section{References}

[1] D. Baratti, S. Kusamura, D. Nonaka, M. Langer, S. An- dreola, M. Favaro, et al., "Pseudomyxoma Peritonei: Clinical Pathological And Biological Prognostic Factors In Patients Treated With Cytoreductive Surgery And Hyperthermic Intraperitoneal Chemotherapy (HIPEC)," Annals of Surgical Oncology, Vol. 15, No. 2, 2008, pp. 526-534. doi:10.1245/s10434-007-9691-2

[2] T. Yan, M. Deraco, D. Baratti, S. Kusamura, D. Elias, O. Glehen, et al., "Cytoreductive Surgery and Hyperthermic Intraperitoneal Chemotherapy for Malignant Peritoneal Mesothelioma: Multi-Institutional Experience,” Journal of Clinical Oncology, Vol. 27, No. 36, 2009, pp. 6237-6242. doi:10.1200/jco.2009.23.9640

[3] T. Chua, T. Yan, A. Saxena and D. Morris, "Should the Treatment of Peritoneal Carcinomatosis by Cytoreductive Surgery and Hyperthermic Intraperitoneal Chemotherapy Still Be Regarded as a Highly Morbid Procedure?: A Systematic Review Of Morbidity And Mortality," Annals of Surgery, Vol. 249, No. 6, 2009, pp. 900-907. doi:10.1097/SLA.0b013e3181a45d86

[4] P. J. Medina, J. M. Sipols and J. N. George, "Drug-AssoCiated Thrombotic Thrombocytopenic Purpura-Hemolytic Uremic Syndrome," Current Opinion in Hematology, Vol. 8, No. 5, 2001, pp. 286-293.

doi:10.1097/00062752-200109000-00004

[5] X. L. Zheng and J. E. Sadler, "Pathogenesis of Thrombotic Microangiopathies," Annual Review of Pathology, Vol. 3, 2008, pp. 249-277.

doi:10.1146/annurev.pathmechdis.3.121806.154311

[6] C. M. Taylor, S. Machin, S. J. Wigmore and T. H. Goodship, "Clinical Practice Guidelines for the Management of Atypical Haemolytic Uraemic Syndrome in the United Kingdom,” British Journal of Haematology, Vol. 148, No. 1, 2010, pp. 37-47.doi:10.1111/j.1365-2141.2009.07916.x

[7] A. Zakarija and C. Bennett, "Drug-Induced Thrombotic Microangiopathy," Seminars in Thrombosis and Hemostasis, Vol. 31, No. 6, 2005, pp. 681-690.

doi:10.1055/s-2005-925474

[8] D. C. Doll, R. B. Weiss and B. F. Issell, "Mitomycin: Ten Years After Approval For Marketing," Journal of Clinical Oncology, Vol. 3, No. 2, 1985, pp. 276-286.

[9] J. B. Lesesne, N. Rothschild, B. Erickson, S. Korec, R. Sisk, J. Keller, et al., "Cancer-Associated Hemolytic-Uremic Syndrome: Analysis Of 85 Cases From A National Registry,” Journal of Clinical Oncology, Vol. 7, No. 6, 1989, pp. 781-789.

[10] N. Ntukidem, C. Arce-Lara, G. A. Otterson, E. Kraut, S. Cataland and T. Bekaii-Saab, "Capped-Dose Mitomycin C: A Pooled Safety Analysis From Three Prospective Clinical Trials," Cancer Chemotherapy and Pharmacology, Vol. 65, No. 2, 2010, pp. 319-324. doi:10.1007/s00280-009-1036-3

[11] R. Shah, E. Beem, L. Sautina, S. I. Zharikov and M. S. Segal, "Mitomycin- and Calcineurin-Associated HUS, Endothelial Dysfunction and Endothelial Repair: A New Paradigm For The Puzzle?,” Nephrology Dialysis Transplantation, Vol. 22, No. 2, 2007, pp. 617-620. doi:10.1093/ndt/gfl586 